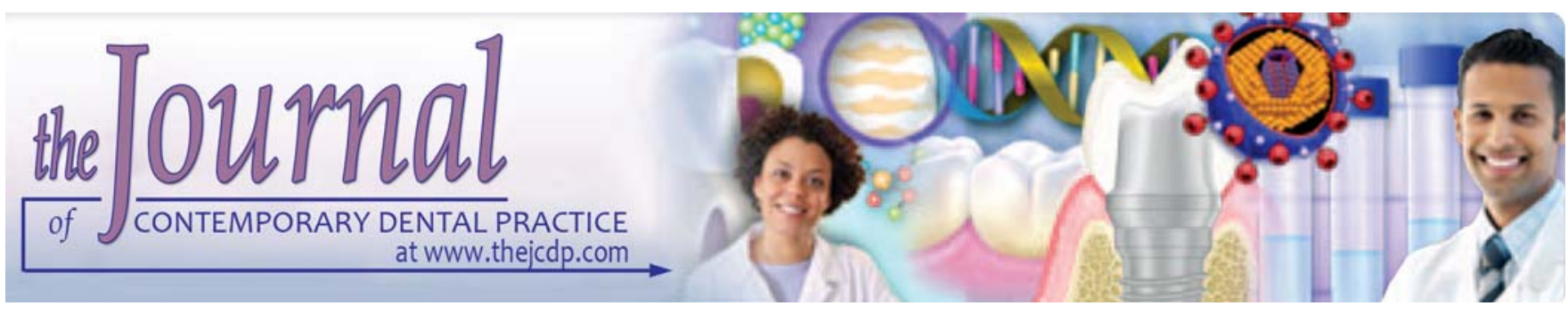

\title{
Analysis of Tumor Marker CA 125 in Saliva of Normal and Oral Squamous Cell Carcinoma Patients: A Comparative Study
}

\author{
Jude J Balan, Roopa S Rao, BR Premalatha, Shankargouda Patil
}

\begin{abstract}
Background: The mortality and morbidity associated with oral squamous cell carcinoma (OSCC) can be greatly reduced if tumor markers which can detect OSCC at an early stage are available. The use of saliva as an alternative to blood could provide a substantial advantage in sampling convenience. Cancer antigen 125 (CA 125) is a tumor-associated antigen found to be increased in epithelial tumors like oral, breast and ovarian cancers.
\end{abstract}

Aim: To determine whether salivary CA 125 levels are increased significantly in OSCC patients than the control group.

Materials and methods: Sixty OSCC patients and 60 healthy controls were taken for the study. Saliva samples from both the groups were collected, centrifuged and supernatant fluid were subjected to ELISA for assessment of CA 125 . The mean salivary CA 125 values of OSCC patients and control group were statistically analyzed using Mann-Whitney U-test.

Results: The mean salivary CA 125 concentration of OSCC group was 320.25 and that of control group was 33.14. Thus, CA 125 was found to be significantly increased in the saliva of OSCC patients than the control group ( $p<0.001)$. Also, there was significant increase in the CA 125 levels as the stage of OSCC increased.

Conclusion: The convenience, reliability and noninvasive nature of salivary CA 125 testing makes it a feasible adjunctive diagnostic tool for detection of OSCC.

Keywords: CA 125, ELISA, Oral squamous cell carcinoma, Saliva, Tumor marker.

How to cite this article: Balan JJ, Rao RS, Premalatha $B R$, $P$ atil S. Analysis of Tumor Marker CA 125 in Saliva of Normal and Oral Squamous Cell Carcinoma Patients: A Comparative Study. J Contemp Dent Pract 2012;13(5):671-675.

\section{Source of support: Nil}

Conflict of interest: None

\section{INTRODUCTION}

Oral cancer refers to all malignancies arising from the lips, oral cavity and pharynx and $90 \%$ of oral cancers are oral squamous cell carcinoma (OSCC). WHO has reported oral cancer as having one of the highest mortality ratios among other malignancies with a 5-year survival rate of $50 \%$. This high mortality rate can definitely be attributed to the late diagnosis of the disease. At the moment, a lack in national screening programs together with a lack of definitive and satisfactory biological markers for early oral cancer detection has resul ted in late stage diagnosis of oral cancer. ${ }^{1}$

Saliva as a diagnostic medium has advanced exponentially over the past few years. In comparison with blood, the collection of saliva is less invasive, painless, inexpensive and safe. ${ }^{2}$ Surprisingly, only few studies have examined tumor markers in the saliva of OSCC patients. Salivary analysis is a useful diagnostic tool for other distant malignancies, such as breast and ovarian cancer. Such an examination might be of great benefit in oral cancer because of the direct contact between the lesion and saliva.

Cancer A ntigen 125 (CA 125) also known as mucin 16 or MUC16 is a protein that in humans is encoded by the MUC16 gene and a member of the mucin family glycoproteins present in the surface cell wall of normal cells. ${ }^{3}$ The antiadhesive property of MUC16 has been suggested to provide a protective barrier for the epithelial surface from bacterial adherence and mechanical injury. Normal levels of CA 125 are found to be in the range of 5 to $80 \mathrm{U} / \mathrm{ml}$. CA 125 is cleaved from the surface of cancer cells and shed into saliva/blood providing a useful tumor marker that may be elevated in patients with epithelial cancers like ovarian, breast and oral carcinoma. ${ }^{4}$ Hence, this study was taken up to assess whether salivary CA 125 can be used to detect OSCC.

\section{MATERIALS AND METHODS}

One hundred and tw enty subjects reporting to M S R amaiah Dental and M edical College and Hospital, Bengaluru were 
selected for the study. Sixty histopathologically diagnosed cases of primary OSCC sixty age and sex method controls were included in the study. Sixty age and sex matched controls were taken. Patients with gingival and mucosal lesions with a tendency to bleed were excluded because leakage of blood into the mouth might be a source of CA 125.

A fter obtaining informed consent, unstimulated saliva was collected by asking the subjects to dribble into sterile glass container. The whole saliva was centrifuged $(3,000$ rpm for 10 minutes) and supernatant collected was subjected to ELISA testing. CA 125 in the samples were quantified using the 'CA 125 quantitative assay kit' (UBI MA GIWEL, California, USA ), which is a solid phase polyclonal enzymelinked immunosorbent assay (Fig. 1).

The samples, standards, controls and biotinylated antiCA 125 antibodies were allowed to incubate in the wells. During the incubation, specific cancer antigen binds to antiCA 125 antibodies on the wells. Unbound CA 125 antigen was removed by washing the wells with buffer. Enzyme conjugate was added to each well. A fter the incubation, unbound enzyme conjugate was washed off and the amount of bound peroxidase will be proportional to the concentration of the CA 125 present in the sample. Upon addition of the chromogen substrate, the intensity of color developed will be proportional to the concentration of CA 125 in the sample and this was quantified by use of a photometric well reader at $450 \mathrm{~nm}$ wavelength (Fig. 2).

The following criteria were used to validate quality control when evaluating results: A zero standard and five reference standards calibrated to 10, 50, 100, 200 and $500 \mathrm{U} / \mathrm{ml}$ were run with each test. The value obtained in the test should be equal to the expected value. If values are not matching, then the test is not valid. $M$ ann-W hitney $\mathrm{U}$-test was used to test the statistical significance of CA 125 values in saliva samples among OSCC patients and

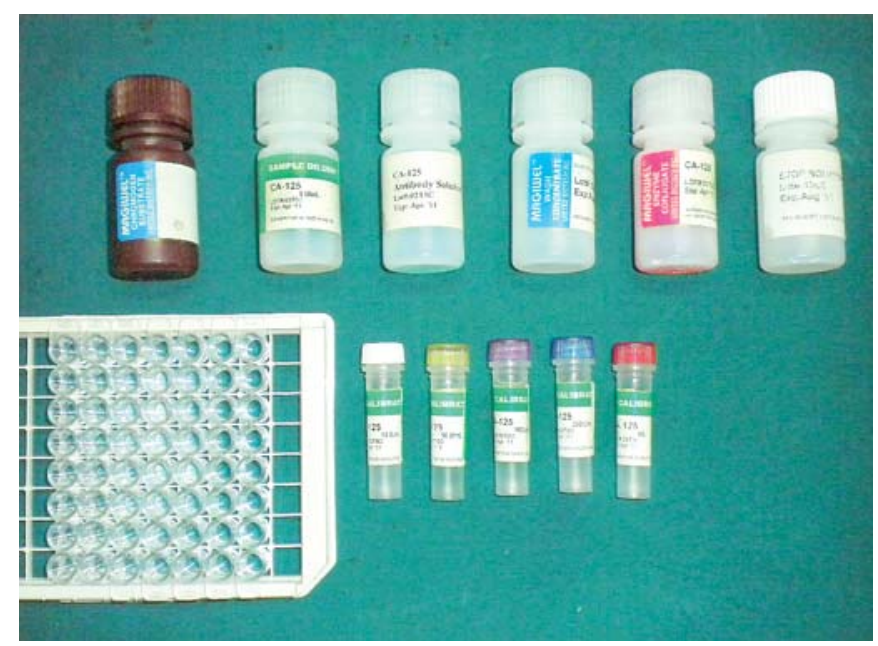

Fig. 1: Armamentarium CA 125 ELISA kit with reagents control subjects. It is the nonparametric variant of unpaired t-test.

\section{RESULTS}

A wide variation in age of the patients was noted, ranging from 23 to 70 years. The mean age was 51.3 years for OSCC patients. The maximum cases were seen in the age group of 41 to 50 years followed by groups aged 51 to 60 years and 61 to 70 years (Table 1). OSCC patients group consisted of 26 male patients and 34 female patients. OSCC patients group consisted of 10, 16, 22 and 12 patients in stages I, II, III and IV respectively (based on TNM staging). Thirty of 60 OSCC patients had metastasis to Iymph nodes, when they reported to the clinician for diagnosis. The most common site of OSCC was buccal mucosa in 24 patients followed by tongue (14), palate (8), labial mucosa (6), floor of the mouth and (4) alveolar ridge (4).

In OSCC patients, the mean salivary CA 125 value was 320.2467 and that of control group was 33.1367 (Graph 1). The p-value was $<0.001$ and thus the mean score of salivary CA 125 levels between OSCC patients and control group was found to be highly significant (Table 2). A mong different stages of OSCC patients, the mean salivary CA 125 value of stage I group was 152.4, stage II group was 207.1, stage III group was 356.6 and stage IV group was 544.2 ( $G$ raph 2). The reliability of saliva for diagnosis of OSCC was measured by calculating sensitivity and specificity. Sensitivity of $84 \%$ and also specificity of $87 \%$ was obtained using $95 \%$ confidence limits.

\section{DISCUSSION}

Despite the advances in the field of surgery and radiotherapy, the treatment outcome of the cancer patients is mostly unsatisfactory. The overall 5-year survival rates for cancers of the tongue, oral cavity and oropharynx are

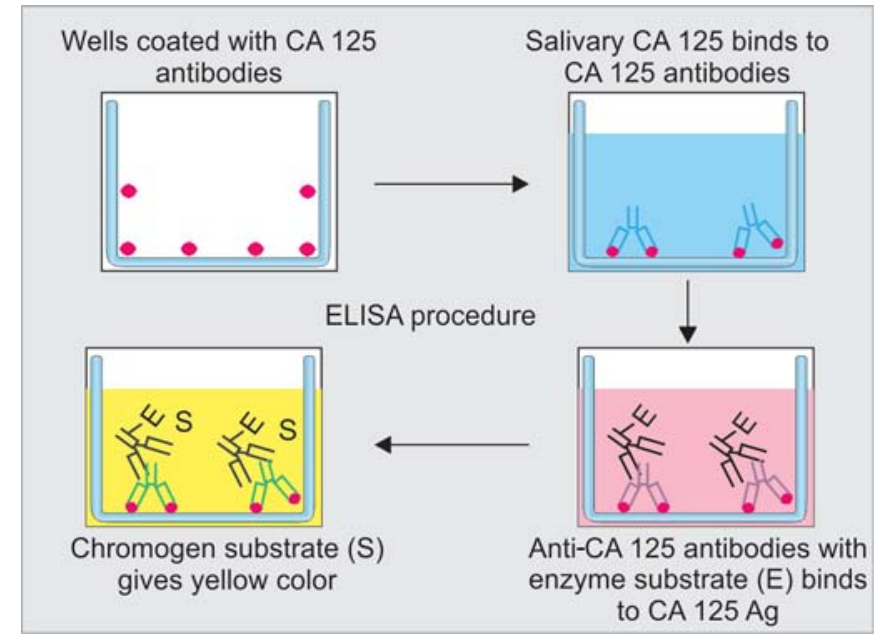

Fig. 2: Schematic diagram of ELISA procedure 
Analysis of Tumor Marker CA 125 in Saliva of Normal and Oral Squamous Cell Carcinoma Patients: A Comparative Study

around 50 to $60 \%{ }^{5,6}$ Poor prognosis for oral cancer is mostly accounted by presentation at a late stage of the disease. Hence, it is important to diagnose oral cancer as early as possible to improve the prognosis necessitating the need

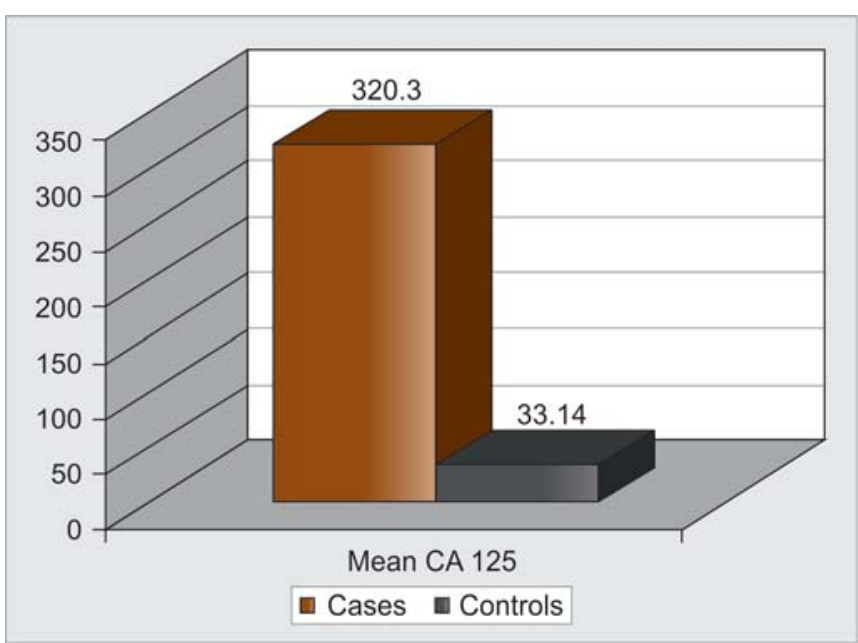

Graph 1: The mean CA 125 levels in OSCC cases and controls

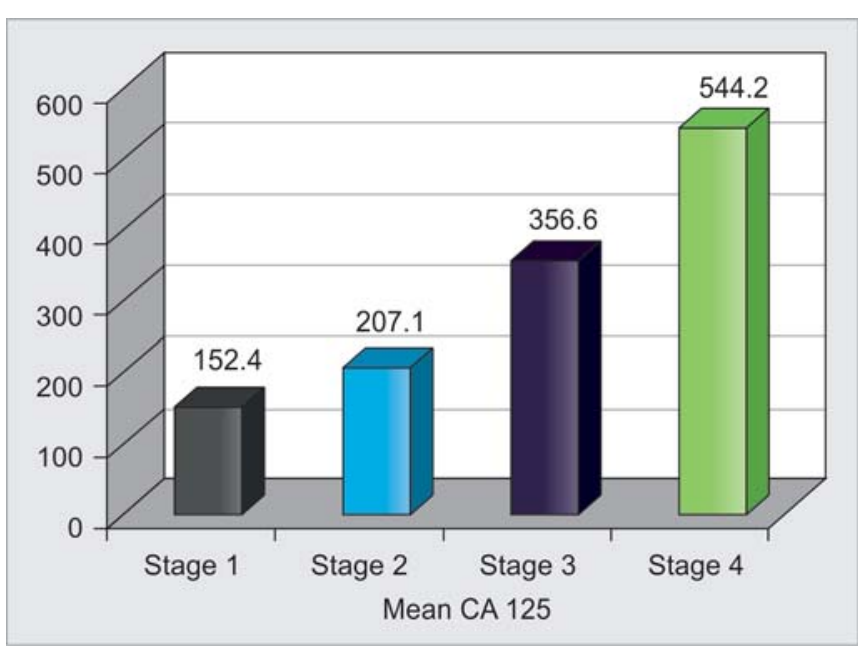

Graph 2: The mean CA 125 levels in different stages of OSCC cases

Table 1: Age and gender-wise distribution of samples

\begin{tabular}{lccccc}
\hline \multirow{2}{*}{ Age } & \multicolumn{2}{c}{ Cases } & & \multicolumn{2}{c}{ Controls } \\
\cline { 2 - 3 } & Male & Female & & Male & Female \\
\hline 21-30 years & 2 & 0 & & 2 & 0 \\
31-40 years & 6 & 6 & & 6 & 6 \\
41-50 years & 6 & 12 & & 6 & 12 \\
51-60 years & 7 & 8 & & 7 & 8 \\
60-70 years & 5 & 8 & & 5 & 8 \\
\hline Total & 26 & 34 & & 26 & 34 \\
\hline
\end{tabular}

for tumor markers which can detect OSCC before metastasi s. $^{7,8}$

In the last few decades, there has been considerable interest in saliva as a safer, more convenient, noninvasive and more easily obtained specimen than blood. Haeckel and Hanecke ${ }^{9}$ and Drobitch ${ }^{10}$ stated that the molecules from blood may reach saliva through various mechanisms like ultrafiltration and transdiffusion without any direct contact between them. In OSCC cases, CA 125 can also reach saliva directly due to increased shedding of cancer cells. Unstimulated whole saliva is preferred over stimulated saliva as it contains detectable quantity of CA 125 . Whole saliva gives consistent results and is more appropriate because it is technically easy to collect, reliable and compliance of the patient serves as an added advantage. It can also be used for mass screening purpose.

The functional role of CA 125/M U C16 in cancer is not well understood. In recent studies by Lakshmanan et al ${ }^{11}$ it was found that CA $125 / \mathrm{MUC16}$ induced rapid G2/M transition causing increased proliferation in breast cancer cells and stable knockdown of M U C 16 in breast cancer cells resulted in significant decrease in the rate of cell growth, tumorigenicity and increased apoptosis. In vitro studies by Comamala and Pinard et al ${ }^{12}$ showed disruption of cellcell junctions, enhanced motility, migration and invasiveness in CA 125/M U C16 knockdown cells. Hence, they suggested that CA 125/M U C 16 plays a role in epithelial mesenchymal transition, presumably through its interaction with $\mathrm{E}$-cadherin and $\beta$-catenin complexes and by modulating EGFR.

All the studies which were done to assess the levels of various tumor markers in saliva of OSCC showed sensitivity ranging from 30 to $92 \%$. N one of the tumor marker showed $100 \%$ sensitivity. There may be various reasons for this lack of accuracy. It may be due to insufficient increase in the tumor marker levels to be detected by low sensitive methods or the particular gene may not be affected in all the patients. To overcome this difficulty, Nagler and Linkov suggested the use of panel of markers instead of single marker, which will increase the sensitivity and specificity. ${ }^{13,14} \mathrm{Although}$ panel of markers might increase the sensitivity and specificity, it is not cost-effective.

In our study, we chose to assess salivary CA 125 because it was reported to be increased in various epithelial tumors like breast, ovarian and oral cancer. ${ }^{15-18} \mathrm{Di}$-Xia et al ${ }^{15}$ and

\begin{tabular}{llccccc}
\multicolumn{7}{c}{ Table 2: Comparison of mean CA 125 values between OSCC patients and control group } \\
\hline CA 125 & $\mathrm{~N}$ & Mean & $\begin{array}{c}\text { Std. } \\
\text { deviation }\end{array}$ & $\begin{array}{c}\text { Minimum } \\
\text { value }\end{array}$ & $\begin{array}{c}\text { Maximum } \\
\text { value }\end{array}$ & p-value \\
\hline SCC cases & 60 & 320.2467 & 208.4518 & 9.20 & 1008.00 & 0.001 \\
Controls & 60 & 33.1367 & 13.1307 & 7.50 & 85.70 & - \\
\hline
\end{tabular}


Plante et al ${ }^{16}$ found that the specificity, diagnostic efficiency and positive predictive value for the saliva assays were better than those for the blood assays of malignant ovarian cancer. They concluded that the determination of salivary CA 125 levels is a feasible, noninvasive approach in the diagnosis of ovarian cancer.

A gha-Hosseini et al ${ }^{17}$ observed that mean saliva and serum CA 125 levels were significantly higher in untreated breast cancer women compared to healthy and treated groups. Furthermore, a salivary CA 125 level was higher than in serum. They concluded that salivary CA 125 can be used to diagnose breast cancer. On literature search, we found only one study for salivary CA 125 in OSCC. Nagler and $B$ ahar et $a^{13}$ analyzed salivary concentration of Cyfra 21-1, TPS, CEA, SCC, CA 125 and CA 19-9 in OSCC patients. They found a significant increase in salivary concentrations of CA 125 in OSCC patients.

In our study, the mean CA 125 concentration of OSCC group was 320.25, and that of control group was 33.14 and the obtained $p$-value was $<0.001$, suggesting that the CA 125 concentration in OSCC patients is significantly higher than the control group. Similar results were found in a study conducted by Nagler and Bahar et al, ${ }^{13}$ where CA 125 levels were increased by $400 \%$ in saliva of OSCC patients than the healthy control group.

In the present study, the mean CA 125 value of stages I, II, III and IV patients is 152.4, 207.1, 356.6 and 544.2 respectively. There is significant increase in salivary CA 125 concentration as the stage of the OSCC increases. This increase may be due to increased shedding of cancer cells in saliva as the tumor enlarges. On literature search, we did not find studies examining CA 125 levels among the various grades of OSCC. But, CA 125 is extensively studied for ovarian cancer and found to correlate with the progression of the disease in $90 \%$ of the cases. ${ }^{19}$ Thus, CA 125 can be used to monitor the disease progression of OSCC also.

The reliability of saliva for diagnosis of OSCC was measured by calculating sensitivity and specificity. The best performance of CA 125 in our study was at a cutoff value of $84 \mathrm{U} / \mathrm{ml}$, with a sensitivity of $84 \%$, and a specificity of $87 \%$. Our study shows higher sensitivity and specificity than a similar study by $\mathrm{N}$ agler and $\mathrm{B}$ ahar et al. This may be attributed to the higher number of stages III and IV patients. In our study, we did not find any significant difference in the concentration of salivary CA 125 between males and females in both OSCC group and control group.

The advent of salivary markers is a valuable adjunctive diagnostic tool for OSCC as it might save many unnecessary biopsies and hospital visits. A Iso, follow-up of treated OSCC patients to detect recurrences might be easy with the help of such markers.

\section{CONCLUSION}

CA 125 levels are found to be increased in saliva of oral cancer, ovarian cancer, breast cancer and other epithelial tumors. Hence, CA 125 can be used as a single marker for various epithelial tumors for screening purposes. The development of such salivary diagnostic tools for these patients is of paramount importance, especially for highrisk populations like patients with premalignant lesions, patients with multiple cancerous lesions, patients with previous history of cancer in general, tobacco and alcohol consumers.

\section{ACKNOWLEDGMENT}

The authors thank Colgate-Palmolive (India) L td. scholarship for their financial assistance in conducting the study.

\section{REFERENCES}

1. Warnakulasuriya S. Global epidemiology of oral and oropharyngeal cancer. Oral Oncol 2009;45:309-16.

2. Streckfus CF, Bigler LR. Saliva as a diagnostic fluid. O ral Dis 2002;8:69-76.

3. Perez BH, Gipson IK. Focus on molecules: Human mucin MUC16. Exp Eye Res 2008;87(5):400-401.

4. Bast RC, Badgwell D, Lu Z, M arquez R, Rosen D. New tumor markers: CA 125 and beyond. Int J Gynecol Cancer 2005;15 (Suppl 3):274-81.

5. Rogers SN, Brown J S, Woolgar JA, Lowe D, M agennis P, Shaw RJ. Survival following primary surgery for oral cancer. Oral Oncol 2009;45:201-11.

6. Gomez I, W arnakulasuriya S, V areta-Centelles PI, LopezJ ornet $P$, Suarez-C unqueiro M, Diz-Dios $P$, et al. Is early diagnosis of oral cancer a feasible objective? Who is to blame for diagnostic delay? Oral Dis 2010;16:333-42.

7. Kujan O, Glenny AM, Duxbury J. Evaluation of screening strategies for improving oral cancer mortality: A cochrane systematic review. J Dent Educ 2005;69:255-65.

8. Sankaranarayanan R, R amadas K, Thomas G, M uwange R, Somanathan $T, M$ athew $B$. Effect of screening on oral cancer mortality in Kerala, India: A cluster randomized controlled trial. L ancet 2005;365:1927-33.

9. Haeckel R, Hanecke P. Application of saliva for drug monitoring: A n in vivo model for transmembranetransport. Eur J Clin Biochem 1996;34:171-91.

10. Drobitch RK, Svensson CK. Therapeutic drug monitoring in saliva. A n update. Clin Pharmacokinet 1992;23:365-79.

11. L akshmanan I, Ponnusamy M P, Das S, Chakraborty S. M U C 16 induced rapid $\mathrm{G} 2 / \mathrm{M}$ transition via interactions with JA K 2 for increased proliferation and anti-apoptosis in breast cancer cells. Oncogene 2012 Feb 16;31(7):805-17.

12. Comamala $M$, Pinard $M$, Thériault $C, M$ atte $I$. Downregulation of cell surface CA 125/MUC16 induces epithelial-tomesenchymal transition and restores EGFR signalling in NIH :OV CA R 3 ovarian carcinoma cells. B J Cancer 2011;104: 989-99.

13. Nagler R, B ahar $G$, et al. Concomitant analysis of salivary tumor markers. A new diagnostic tool for oral cancer. Clin Cancer Res 2006;12(13):3979-84. 
14. Linkov F, Lisovich A, Y urkovetsky Z. Early detection of head and neck cancer: Development of a novel screening tool using multiplexed immunobead-based biomarker profiling. Cancer Epidemiol. Biomarkers Prev 2007;16(1):102-07.

15. Di-X ia, Chen, Schwart, Peter E, Fan-Q in Li. Saliva and serum CA 125 assays for detecting malignant ovarian tumors. Obstet Gynecol 1990 A pr;75(4):701-04.

16. Plante $M$, Wong GY, Nisselbaum JS. Relationship between saliva and serum CA 125 in women with and without epithelial ovarian cancer. O bstet Gynecol 1993 J un;81(6):989-92.

17. Agha-Hosseini F, M irzaii-Dizgah I, Rahimi A. Correlation of serum and salivary CA 125 levels in patients with breast cancer. J Contemp Dent Pract 2009 Nov 1;10(6):E001-08.

18. Tay SK, Chua EK. Correlation of serum, urinary and salivary CA 125 levels in patients with adnexal masses. A nn A cad M ed Singapore $1994 \mathrm{M}$ ay;23(3):311-14.

19. Hunter VJ, Daly L, Helms $M$. The prognostic significance of CA 125 half-life in patients with ovarian cancer who have received primary chemotherapy after surgical cytoreduction. A m J Obstet Gynecol 1990;163:1164-67.

\section{ABOUT THE AUTHORS}

\section{Jude J Balan (Corresponding Author)}

Postgraduate Student, D epartment of Oral Pathology, MS Ramaiah Dental College and Hospital, Bengaluru, Karnataka, India e-mail: jjudeib@gmail.com

\section{Roopa S Rao}

Professor and Head, Department of Oral Pathology, MS Ramaiah Dental College and Hospital, B engaluru, Karnataka, India

\section{BR Premalatha}

Senior L ecturer, Department of Oral Pathology, M S Ramaiah D ental College and Hospital, B engaluru, K arnataka, India

\section{Shankargouda Patil}

Senior L ecturer, Department of Oral Pathology, M S Ramaiah D ental College and Hospital, B engaluru, K arnataka, India 